\title{
A test for S-S associations in a conditional counting task
}

\author{
RICHARD A. BURNS and FRANCES A. NESBITT \\ Georgia Southwestern College, Americus, Georgia
}

\begin{abstract}
Conditional numerical discriminations were established in runway-trained rats by presenting daily a pair of reward series that differed in the number of rewarded trials preceding a terminal nonrewarded trial. One kind of series contained three rewarded trials (RRRN or NRRRN), which always occurred in either a smooth, black runway or a rough, white runway (counterbalanced). The other series contained four rewarded trials (RRRRN or NRRRRN), which occurred in a runway of the other brightness and texture. The reward on each rewarded trial was $5.045-\mathrm{g}$ Noyes pellets until Day 22. For Days 22-32, the series containing four Rs was altered so that the number of pellets on each respective trial was $14,7,3$, and 1 . To test whether S-S associations controlled the serial discriminations after Day 32, we rearranged the order of elements in the 14-7. 3-1-0 series to 20-7-3-14-0. Rapid approach to the terminal series element on the test day would have implied the operation of S-S associations (14 pellets would have been a cue for upcoming 7 , not 0 ), but the approach to that element remained slow, suggesting that the animals continued their counting of rewarded trials during the training with the S-S-relevant 14-7-3-1-0 series, irrespective of the order of the series elements.
\end{abstract}

A classic childhood riddle asks, "How many fingers do you have?" and the right answer requires that the digits by categorized in two subordinate classes within which the instances are counted separately. The usual answer, funny to children, ignores the categories.

Some new experiments imply that problems similar to the children's riddle can be mastered by rats. Runwaytrained rats given the series $R^{\prime} R R N$, where $R^{\prime}$ and $R$ are two different reward outcomes presented sequentially and $\mathrm{N}$ is a terminal nonreward, learn to run slowly only on the last trial of the series; and they transfer well to either the RRN or the RRRN series (Burns \& Gordon, 1988; Capaldi \& Miller, 1988). The riddle taps several processes. For the rat to transfer to both the RRN (2R) and the RRRN (3R) series, the initial $R^{\prime} R R N$ series must have been categorized; concurrent counting must have taken place within each subordinate category, two Rs and one $\mathrm{R}^{\prime}$; and a general counting of reward events in the superordinate category, "rewards," must have taken place, three Rs. Superordinate counting alone would have the rats transferring well only to the $3 R$ test series-the funny answer.

That rats are capable of concurrently counting different numbers of rewarded trials has been indicated in several conditional counting experiments (Burns, Nesbitt, Cribb, \& Hulbert, in press; Burns \& Sanders, 1987; Capaldi, Miller, \& Alptekin, 1989) as well as in the cate-

This work was supported by a grant from the Charles L. Mix Foundation to Richard A. Burns. The authors thank Charles J. Knighton for his assistance. Address correspondence to Richard A. Burns, Department of Psychology, Georgia Southwestern College, Americus, GA 31709. BITNET: RBURNS@UGA. gorical counting experiments just described. One of our purposes in the work to be reported here was to continue the examination of numerical processes involved in categorical and conditional counting investigations by studying a conditional discrimination based on three and four rewarded trials, our other work having involved only one, two, or three events to be counted.

In any serial learning task, counting the elements of the series is one means by which an animal may come to anticipate those elements. The series 14-7-3-1-0, for instance, is a series of differing numbers of pellets given as reward in many serial learning studies (e.g., Hulse \& Dorsky, 1977; Roitblat, Pologe, \& Scopatz, 1983). One way rats could anticipate the terminal nonrewarded element of that series, and run slowly to it, would be to count the rewarded trials without categorizing them on the basis of reward amount. The chore, if that were done, would be a $4 \mathrm{R}$ problem and nothing more.

Transfer from training on a 14-7-3-1-0 series to a 207-3-14-0 series was examined in an experiment reported by Haggbloom (1985), who found that the slower running to the terminal element of the training series did not transfer to the test series. Those findings strongly imply that $\mathrm{S}-\mathrm{S}$ associations were the basis of learning the serial anticipation (see Capaldi, 1985). After conditional numerical discriminations of three and four rewarded trials were established in the experiment we will describe here, we modified the $4 R$ series so that it became a 14-7-3-1-0 series. Because we used 5 pellets on all rewarded trials during the $3 R / 4 R$ training, this shift was from a numberrelevant, S-S-irrelevant to a number-relevant, S-Srelevant problem. The purpose was to see if training on a number-relevant task would block the subsequent de- 
velopment of S-S associations. The test for control by S-S associations was the test used by Haggbloom (1985), a shift to 20-7-3-14-0.

\section{METHOD}

\section{Subjects}

The subjects were 4 naive male rats purchased from the Holtzman Company, Madison, WI. The animals were about 70 days old at the beginning of training.

\section{Apparatus}

The apparatus was the same L-shaped, black wooden runway that we have used in two previous conditional counting investigations (Burns et al., in press; Burns \& Sanders, 1987). A hinged white panel made of aluminum truck bumper stock with a raised diamond pattern allowed ready modification of the runway floor from smooth and black to rough and white. The time for the animal to travel the first $195.6 \mathrm{~cm}$ of the runway after the opening of the start door was digitally recorded in units of $.01 \mathrm{sec}$.

\section{Procedure}

The animals were housed in a naturally lighted, windowed colony room in individual living cages with continuous access to water. For 2 days after their arrival, the rats were allowed free access to food ( $\mathrm{Pu}$ rina Rodent Chow), and for the remainder of the experiment they were given $12 \mathrm{~g}$ of chow on each day that preceded a training day. They were trained only on weekdays, and they were given $18 \mathrm{~g}$ of chow when they were not to be trained the following day. At the experiment's conclusion, the animals were released into our outdoor retirement colony (Burns \& Fort, in press).

After the initial days of free feeding, the animals were allowed a daily 15-20 min period together on a walled exercise table prior to being returned to their cages, which contained the food ration. This practice was maintained throughout the experiment, and it followed the day's training on all days except the first 3, during which the rats were handled and nothing more. There followed 3 days of goal area placements. Each rat was placed in the baited goal area with the retrace door closed and given up to $5 \mathrm{~min}$ to consume the reward (five .045-g Noyes pellets). There were five such placements for each rat on each of 3 days, and the runway floor alternated from smooth and black to rough and white with each placement.

Primary experimental training consisted of presenting to each rat two different series containing both rewarded and nonrewarded trials. All series ended in nonreward. On each day, one series contained three successive rewarded trials (a 3R series) and the other contained four successive rewarded trials (a 4R series). On odd days, the 3R series began with an initial nonreward and the 4R series did not (NRRRN/RRRRN); and on even days, the $4 R$ series began with nonreward (RRRN/NRRRRN). For 2 of the rats, all 3R series appeared in the conditional context of a smooth, black runway, and all $4 R$ series appeared in the rough, white runway. For the remaining 2 animals, the relationship between number and context was reversed. Each day the order of running of the animals as well as the order of presentation of the series was determined randomly. The interval between the trials of a series was about $20 \mathrm{sec}$, because each rat was presented all the trials of a series in succession before another was run. The interval between series was about $18 \mathrm{~min}$, because the second series of the day was not begun until all animals had completed their first series. A trial began with the opening of the start door about $2 \mathrm{sec}$ after placement of the animal in the start area, and it terminated after the reward (five pellets) was consumed or after $30 \mathrm{sec}$ in an unbaited goal area on nonrewarded trials.

Data for the first day of primary training were actually collected over a 2-day period, because only one series was run on each of those days to compensate for the prolonged running times that occur early in training. After 21 days of primary training, stable numerical discriminations had become apparent, and the reward values in the $4 R$ series were changed

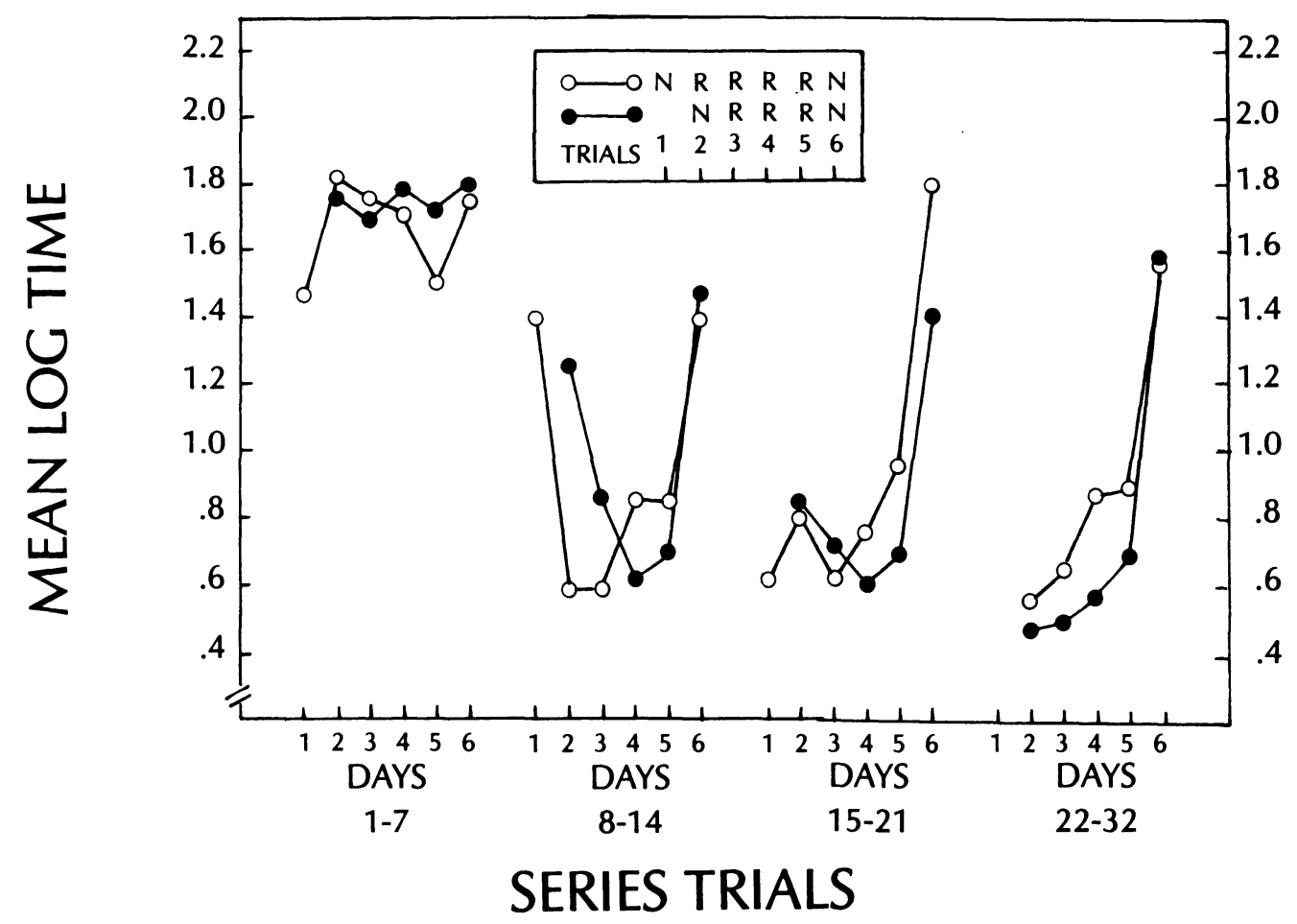

Figure 1. Average transformed running times toward each element in the 3R and $4 R$ series during Days 1-21, in blocks of 7 days. The last trial of each series is depicted as Trial 6, regardless of the actual number of trials in the series. The figure also plots performance from Day 22 through Day 32, during which the $4 \mathrm{R}$ series contained the elements 14-7-3-1-0. 
from 5 pellets on each rewarded trial (5-5-5-5-0) to 14-7-3-1-0. Training continued on the 14-7-3-1-0 series, and, of course, on the unchanged 3R series, until Day 33, when the elements of the $4 R$ series were once again changed and also rearranged to $20-7-3-14-0$ as a test for the effects of S-S associations.

\section{RESULTS}

All running times were transformed $[x=\log (x)]$ for statistical analyses. Plotted in Figure 1 are the average running times on each trial of the $3 R$ and $4 R$ series over the first 21 days in blocks of 7 days. Also in Figure 1 is the plot over trials of the unmodified $3 R$ series and the $4 \mathrm{R}$ series that consisted of the elements 14-7-3-1-0, which were examined on Days 22-32. The terminal nonrewarded trial is always depicted as Trial 6 , irrespective of its actual trial position.

That these animals came to run more slowly toward the terminal, nonreward, element of both the $3 R$ and $4 R$ series is conspicuous in Figure 1. To statistically evaluate the numerical discriminations in series that differed in number of trials, the initial outcomes of which were made unpredictable, we performed repeated measures analyses of variance that included only the last four trials of the two series. A reliable main effect of trials $[F(3,9)=$ $12.42, p<.01$ ] on Days 8-14 was evaluated using the Bonferroni $t$ statistic $[k(9)=6]$, which showed that approach to the terminal series element was reliably slower than to the three preceding elements, each of which did not differ from the other. There was no clear difference in the way the animals approached the $3 \mathrm{R}$ and the $4 \mathrm{R}$ se-

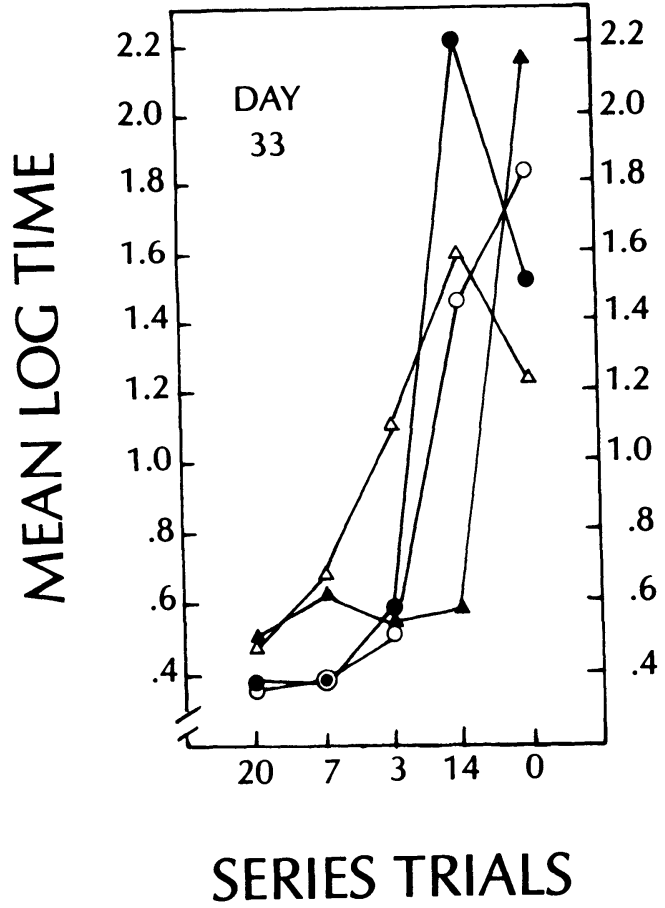

Figure 2. Mean running time for each of the 4 animals during the single test day, Day 33, with the modified series 20-7-3-14-0. ries $[F(3,9)=3.76, p>.05]$. The pattern of results established for Days 8-14 was evident also in the analysis of Days $15-21[F(3,9)=9.14, p<.01]$ and Days $22-32$ $[F(3,9)=94.10, p<.01]$.

In Figure 2, the running of each of the 4 rats on the Day 33 test series, 20-7-3-14-0, is illustrated. The animal's approach in this series to the terminal element was once again significantly slower than it was to the initial, rewarded series elements $[F(4,12)=7.49, p<.01]$, but the Bonferroni comparisons among the main effect means $[k(12)=10]$ showed that running to the reward immediately preceding the terminal nonreward was also slower-it had not been so before-than running to the two initial rewards.

\section{DISCUSSION}

Conditional discriminations of two and three rewarded trials were reported by Burns and Sanders (1988) and by Burns et al. (in press). The discriminations we found here under similar training conditions, but with three and four rewards to count, were generally comparable to those reported earlier. Apparently number is a salient dimension to which rats readily attend. These and other findings work against the view held by Davis and Perusse (1988) that attention to the number of things is only a last resort. It seems evident too that number may be conditionally discriminated by rats just as other stimuli can, and that conditional numerical discriminations are a means by which number may become associated with otherwise nonnumerical things as, for example, when we learn that uno, un, jedan, or ein mean "one," and dos, deux, dva, or zwei mean "two."

When Haggbloom (1985) looked for transfer from a 14-7-3-1-0 series to a 20-7-3-14-0 series, he found none. His animals ran equally fast on all of the trials in the transfer series after having developed a strong tendency to run slowly to the terminal element of the training series. The results of our shift were different. Most important was that running slowly to the terminal element persisted in the transfer series. This finding implies that $\mathrm{S}-\mathrm{S}$ associations did not control the discriminations in the 14-7-3-1-0 series. The view that noncategorical counting of rewarded trials carried over from the original conditional counting, number-relevant training would predict ready transfer to the 20-7-3-14-0 series, and that is what happened. A clear tendency to run slowly on the trial preceding the terminal nonreward in the transfer series cannot, however, be readily understood by means of reference to counting.

\section{REFERENCES}

BURNS, R., \& FORT, M. (in press). An outdoor retirement village for experienced laboratory rats. Humane Innovations \& Alternatives in Animal Experimentation.

BuRNs, R. A., \& GoRDON, W. U. (1988). Some further observations on serial enumeration and categorical flexibility. Animal Learning \& Behavior, 16, 425-428.

Burns, R. A., Nesbitt, F. A., Cribb, D., \& Hulbert, L. G. (in press). Conditional discrimination of series containing either two or three rewarded trials. Journal of General Psychology.

Burns, R. A., \& SANDERS, R. E. (1987). Concurrent counting of two and three events in a serial anticipation paradigm. Bulletin of the Psychononic Society, 25, 479-481.

CAPAlDI, E. J. (1985). Anticipation and remote associations: A configural approach. Journal of Experimental Psychology: Learning, Memory, \& Cognition, 11, 444-449.

CaPaldi, E. J., \& Miller, D. J. (1988). Counting in rats: Its functional significance and the independent cognitive processes which comprise it. Journal of Experimental Psychology: Animal Behavior Processes, 14, 3-17.

Capaldi, E. J., Miller, D. J., \& Alptekin, S. (1989). A conditional numerical discrimination based on qualitatively different reinforcers. Learning \& Motivation, 20, 48-59. 
Davis, H., \& Perusse, R. (1988). Numerical competence in animals: Definitional issues, current evidence, and a new research agenda. $B e$ havioral \& Brain Sciences, 11, 561-615.

HAGGBLOOM, S. J. (1985). Serial learning and transfer in rats: Effects of changes in stimulus-stimulus associations, pattern structure, and serial position information. Animal Learning \& Behavior, 13, 370-374.

Hulse, S. H. , \& DorsKy, N. P. (1977). Structural complexity as a de- terminant of serial pattern learning. Learning \& Motivation, 8, 488-506.

Roitblat, H. L., Pologe, B., \& Scopatz, R. A. (1983). The representation of items in serial position. Animal Learning \& Behavior, 11, 489-498.

(Manuscript received March 8, 1990.) 